(c) 2005 International Press

Adv. Theor. Math. Phys. 9 (2005) 527-558

\title{
Black holes and the spectrum of half-BPS states in $N=4$ supersymmetric string theory
}

\author{
Ashoke Sen \\ Harish-Chandra Research Institute, Chhatnag Road, Jhusi, \\ Allahabad 211019, India \\ ashoke.sen@cern.ch or sen@mri.ernet.in
}

\begin{abstract}
The entropy of a half-BPS black hole in $N=4$ supersymmetric heterotic string compactification is independent of the details of the charge vector and is a function only of the norm of the charge vector calculated using the appropriate Lorentzian metric. Thus, in order for this to agree with the degeneracy of the elementary string states, the latter must also be a function of the same invariant norm. We show that this is true for generic CHL compactifications to all orders in a power series expansion in the inverse charges, but there are exponentially suppressed corrections which do depend on the details of the charge vector. This is consistent with the hypothesis that the black hole entropy reproduces the degeneracy of elementary string states to all orders in a power series expansion in the inverse charges, and helps us extend the earlier conjectured relation
\end{abstract}

e-print archive: http://lanl.arXiv.org/abs/hep-th/0504005 
between black hole entropy and degeneracy of elementary string states to generic half-BPS electrically charged states in generic $N=4$ supersymmetric heterotic string compactification. Using this result, we can also relate the black hole entropy to the statistical entropy calculated using an ensemble of elementary string states that contains all BPS states along a fixed null line in the lattice of electric charges.

\section{$1 \quad$ Introduction and summary}

Recent progress [1-7] towards relating the degeneracy of half-BPS states in the spectrum of elementary string $[8,9]$ to the entropy of the black hole carrying the same charge quantum numbers [10-12] opens up the possibility of exploring the relationship between the black hole entropy and the statistical entropy in string theory in much more detail than has been achieved so far. In carrying out a similar analysis for systems involving D-branes and other non-perturbative objects in string theory $[13,14]$, one encounters the problem that while it is relatively easy to compute the degeneracy of BPS states in the limit of large charges, it is not always possible to make a more precise estimate that is valid even for finite values of the various charges. In contrast, the degeneracy of elementary string states can be calculated precisely in a wide variety of theories in which the string world-sheet theory is described by a solvable conformal field theory. This in turn should make it possible to carry out a detailed comparison of this degeneracy with the black hole entropy that goes beyond the large charge limit.

However, in carrying out this programme, we encounter a conceptual difficulty: finite size corrections to entropy (and other thermodynamic quantities) depend on the ensemble that we use, and hence there is no unambiguous expression for the statistical entropy even if we know the spectrum exactly. This is due to the fact that in order to establish the equivalence between different ensembles we need to replace the ensemble average of any quantity by the value of the quantity for the most probable configuration. This is a valid approximation in the infinite size limit where the probability distribution is sharply peaked around the most probable configuration, but is not strictly valid for a finite size system. Thus, in order to carry out a comparison between the entropy of a black hole to the degeneracy of elementary string states beyond the large charge limit, we first need to decide which ensemble we should use to compute the statistical entropy of elementary string states. Since there is as yet no fundamental principle that determines this, we can pose the question in a slightly different way: is there a statistical ensemble for half-BPS elementary string states such that the 
entropy computed using this ensemble agrees with the entropy of the black hole carrying the same charge quantum numbers?

So far there has been two different proposals for the choice of ensembles. The first one uses an expression for the black hole entropy computed without taking into account the effect of holomorphic anomaly in the expression for the generalized prepotential and relates it to the statistical entropy computed using an ensemble where we keep half of the charges ("magnetic charges") fixed and sum over all possible values of the other charges after introducing chemical potential conjugate to these charges $[1,7,15,16]$. The second proposal uses an expression for the black hole entropy computed after taking into account the effect of holomorphic anomaly [17-19] following [20], and relates it to the statistical entropy of an ensemble where a single T-duality invariant combination of the charges is allowed to vary $[3,6]{ }^{1}$ The relationship between these two proposals is not completely clear at present.

In this paper, we continue our study of the second proposal. We find that in order to generalize this proposal to generic half-BPS states in a generic $\mathcal{N}=4$ supersymmetric heterotic string compactifications, the spectrum of half-BPS states in these theories must satisfy some consistency conditions. In particular, the degeneracy should be expressible as a continuous function of the charges and should not jump by "large" amount as we move from one point on the charge lattice to a neighbouring point, even if the two charges arise from different twisted sectors of an orbifold theory. We then go ahead and analyze the spectrum of half-BPS states in these theories to show that these consistency conditions are satisfied, and furthermore that the spectrum agrees with the proposed formula for relating the black hole entropy to the statistical entropy of half-BPS elementary string states up to exponentially suppressed correction terms. We also give an alternative interpretation of the ensemble used in computing the statistical entropy. In this interpretation, the ensemble includes all states whose charges lie along a fixed null line in the lattice of physical electric charges.

Since part of the paper is somewhat technical, we shall now summarize the main results in some more detail. Let us consider an $\mathcal{N}=4$ supersymmetric heterotic string compactification [22-27] with $(22-k)$ matter multiplets. This theory has a rank $(28-k)$ gauge group, and a generic eletrically charged state is labelled by a $(28-k)$-dimensional electric charge vector. There is a natural T-duality invariant inner product of signature $(22-k, 6)$ in the $(28-k)$-dimensional lattice of electric charges. Let us

\footnotetext{
${ }^{1} \mathrm{~A}$ different version of this ensemble for studying entropy of quarter BPS black holes have been suggested in [21].
} 
consider a black hole solution in this theory carrying electric charge vector $Q$, and let $N=Q^{2} / 2$, where $Q^{2}$ denotes the $S O(22-k, 6)$ invariant norm of this charge vector. It turns out that $S_{\mathrm{BH}}$ is a function of $N$ alone and does not depend on the details of the vector $Q$. We now define $\mathcal{F}_{\mathrm{BH}}(\mu)$ as the Legendre transform of $S_{\mathrm{BH}}(N)$ :

$$
\mathcal{F}_{\mathrm{BH}}(\mu)=S_{\mathrm{BH}}(N)-\mu N,
$$

where $N$ is the solution of the equation

$$
\frac{\partial S_{\mathrm{BH}}(N)}{\partial N}-\mu=0
$$

The proposed relation between the degeneracy $d(Q)$ of elementary string states carrying charge $Q$ and $\mathcal{F}_{\mathrm{BH}}(\mu)$ is ${ }^{2}$

$$
d(Q) \simeq \frac{1}{2 \pi i} \mathrm{e}^{-C_{0}} \int_{\mu_{0}-i a}^{\mu_{0}+i a} d \mu \mathrm{e}^{\mathcal{F}_{\mathrm{BH}}(\mu)+\mu N},
$$

where $a$ is a sufficiently small but fixed positive number, but is otherwise arbitrary, $\mathrm{e}^{-C_{0}}$ is an overall normalization constant whose value we are unable to determine due to an uncertainty in computing the additive constant in the expression for $S_{\mathrm{BH}}$, and $\mu_{0}$ is the saddle point of the integrand on the positive real axis, corresponding to the solution of the equation:

$$
\mathcal{F}_{\mathrm{BH}}^{\prime}\left(\mu_{0}\right)+N=0, \quad \operatorname{Im}\left(\mu_{0}\right)=0, \quad \operatorname{Re}\left(\mu_{0}\right)>0 .
$$

The $\simeq$ in equation (1.3) denotes equality up to error terms which are suppressed relative to the leading term by powers of $\mathrm{e}^{-\pi \sqrt{N}}$. As long as $a$ is sufficiently small, the contribution to equation (1.3) is dominated by the saddle point of the integrand given in equation (1.4), and the result of doing the integration is independent of $a$ up to terms that are suppressed by powers of $\mathrm{e}^{-\pi \sqrt{N}}$. If we simply replace the integral by the value of the integrand at the saddle point, the right-hand side of equation (1.3) reduces to the exponential of the inverse Legendre transform of $\mathcal{F}_{\mathrm{BH}}(\mu)$ up to a multiplicative constant. This gives us back the usual leading order relation $\ln d(Q)=S_{\mathrm{BH}}$ up to an additive constant. However, equation (1.3) is a refined version of this proposal which is supposed to reproduce $\ln d(Q)$ to all orders in a power series expansion in $1 / N$. One of the consequences of the proposal given in equations (1.1)-(1.3) is that if we ignore corrections to $d(Q)$ which are suppressed by powers of $\mathrm{e}^{-\pi \sqrt{N}}$, then $d(Q)$ must be a function $d_{N}$ only of the combination $N=Q^{2} / 2$ and should not depend on the details of the charge vector $Q$.

\footnotetext{
${ }^{2}$ As usual, in computing $d(Q)$, we sum over all angular momentum states.
} 
Explicit computation of the black hole entropy shows that the part of $\mathcal{F}_{\mathrm{BH}}(\mu)$ that contributes to equation (1.3) up to terms suppressed by powers of $\mathrm{e}^{-\pi \sqrt{N}}$ has the form

$$
\mathcal{F}_{\mathrm{BH}}(\mu) \simeq \frac{4 \pi^{2}}{\mu}+\frac{24-k}{2} \ln \frac{\mu}{2 \pi}+C,
$$

where $C$ is a constant whose value is not known at present. Substituting this into equations (1.3) and (1.4), we get

$$
d(Q) \simeq \frac{\mathrm{e}^{C-C_{0}}}{2 \pi i} \int_{\mu_{0}-i a}^{\mu_{0}+i a} d \mu \exp \left(\frac{4 \pi^{2}}{\mu}+\frac{24-k}{2} \ln \frac{\mu}{2 \pi}+\mu N\right)
$$

and

$$
-\frac{4 \pi^{2}}{\mu_{0}^{2}}+\frac{24-k}{2 \mu_{0}}+N=0
$$

Note that $\mu_{0}$ calculated from equation (1.7) is a function of $N$, but we shall avoid displaying this dependence explicitly in order to avoid cluttering up the various formulæ.

The proposed relation (1.3) differ from that in $[1,7,15,16]$ in two important ways. First of all the black hole entropy is computed using the S-duality invariant one particle irreducible effective action that includes explicit nonholomorphic corrections to the generalized prepotential instead of the Wilsonian effective action where such non-holomorphic corrections are absent. Second, the Laplace transform in equation (1.3) is taken with respect to a single variable $\mu$ conjugate to the combination $N=Q^{2} / 2$ instead of the chemical potentials conjugate to all the electric charges.

In section 2, we shall review the result for $S_{\mathrm{BH}}(N)$ that leads to equation (1.5). We also give various different (but equivalent) versions of the proposal (1.3). For example, we show that equation (1.3) may be re-expressed as

$$
\mathcal{F}_{\mathrm{BH}}(\mu) \simeq \mathcal{F}(\mu)+C_{0}
$$

where

$$
\exp (\mathcal{F}(\mu)) \equiv \frac{1}{M} \sum_{N} d_{N} \mathrm{e}^{-\mu N} .
$$

Here, $d_{N}$ is the degeneracy of elementary string states with $Q^{2}=2 N$ and $M$ is an integer that counts the number of allowed values of $N$ per unit interval. $\simeq$ in equation (1.8) denotes equality up to terms suppressed by powers of $\mathrm{e}^{-\pi^{2} / \mu}$. The sum over $N$ in equation (1.9) runs over all allowed values of $N$ in the theory. In computing $d_{N}$ on the right-hand side of equation (1.9), we must pick a specific representative charge vector $Q$ satisfying $Q^{2}=2 N$ and identify $d_{N}$ as the degeneracy of these states. Different representatives differ from each other by terms suppressed by powers of $\mathrm{e}^{-\pi \sqrt{N}}$ which introduces 
an uncertainty in $\mathcal{F}(\mu)$ that is suppressed by powers of $\mathrm{e}^{-\pi^{2} / \mu}$. This does not affect our analysis of the proposed relation equation (1.8) since this relation is expected to be valid in the small $\mu$ limit up to exponentially suppessed terms.

Taking the inverse Legendre transform on both sides, we may also express equation (1.8) as:

$$
S_{\mathrm{BH}}(N) \simeq \widetilde{S}_{\text {stat }}(N)+C_{0},
$$

where $\widetilde{S}_{\text {stat }}(N)$ is the "statistical entropy" of half-BPS elementary string states defined through the relation:

$$
\widetilde{S}_{\text {stat }}\left(N^{\prime}\right)=\mathcal{F}(\mu)+\mu N^{\prime}, \quad \frac{\partial \mathcal{F}(\mu)}{\partial \mu}+N^{\prime}=0 .
$$

$\simeq$ in equation (1.10) denotes equality up to error terms which are suppressed by powers of $\exp (-\pi \sqrt{N})$.

In section 3, we shall verify the proposal (1.3) relating black hole entropy and degeneracy of elementary string states by computing the latter in $\mathcal{N}=4$ supersymmetric heterotic string compactifications. In particular, verification of equation (1.3) requires proving that up to correction terms suppressed by powers of $\mathrm{e}^{-\pi \sqrt{N}}$, the result for $d(Q)$ depends only on the combination $Q^{2}$ and not on the details of the charge vector $Q$. The functional dependence on $Q^{2}$ (including the overall normalization) must be the same irrespective of whether the state arises in the untwisted sector or one of the twisted sectors of the theory.

In the form given in equations (1.8)-(1.10), the proposed relations are equivalent to identifying the black hole entropy to the entropy of half-BPS elementary string states in an ensemble that contains BPS states of different charges, counting all the states with a given $Q^{2}$ value $2 N$ as one state. $\mu$ denotes the chemical potential conjugate to the variable $N=Q^{2} / 2$. This looks a bit odd since $Q^{2}$ is a quadratic function of the charges and hence is not an additive quantum number. A more natural choice of an ensemble would be one in which we keep some charges fixed, introduce a chemical potential conjugate to the other charges, and sum over all possible values of the other charges. We demonstrate in section 4 that such an interpretation is possible for the partition function defined in equation (1.9). We take two fixed charge vectors $Q_{0}$ and $s_{0}$ on the lattice of physical electric charges with $s_{0}$ being null, and consider an ensemble that contains all states of the form $Q_{0}+n s_{0}$ with $n \in Z$. If $\beta$ denotes the chemical potential conjugate to the integer $n$, then the partition function $\exp \left(\widehat{\mathcal{F}}\left(Q_{0}, s_{0}, \beta\right)\right)$ of this ensemble has a simple relation to the partition function $\exp (\mathcal{F}(\mu))$ introduced in equation (1.9). Thus, the proposed relation (1.10) may also be regarded 
as the statement of equivalence between the black hole entropy and the statistical entropy associated with an ensemble of elementary string states whose charges lie along a null line in the lattice.

\section{Entropy of half-BPS black holes and its relation to the degeneracy of elementary string states}

Let us consider an $\mathcal{N}=4$ heterotic string compactification with $(22-k)$ massless matter multiplets [22-27]. This theory has altogether $(28-k)$ $\mathrm{U}(1)$ gauge fields, of which six are graviphoton fields arising out of the right-moving currents on the world-sheet and $(22-k)$ are part of the matter multiplets arising out of the left-moving currents on the world-sheet. We shall refer to these gauge fields as right-handed and left-handed gauge fields, respectively. We consider a half-BPS black hole carrying $(28-k)$ dimensional electric charge vector $Q$. There is a natural Lorentzian metric of signature $(22-k, 6)$ in the vector space of the charges. We define

$$
N=\frac{Q^{2}}{2}
$$

where $Q^{2}$ is the norm of $Q$ measured with this metric. At any given point, in the moduli space of the theory, the $(28-k)$-dimensional vector space has a natural decomposition into a direct sum of a $(22-k)$-dimensional vector space of left-handed electric charges and a six-dimensional vector space of right-handed electric charges. The latter charges couple to the graviphoton fields. If we denote by $\vec{Q}_{\mathrm{L}}$ and $\vec{Q}_{\mathrm{R}}$ the left and the right-handed components of the charges, then

$$
Q^{2}=\left(\vec{Q}_{\mathrm{R}}^{2}-\vec{Q}_{\mathrm{L}}^{2}\right), \quad N=\frac{1}{2}\left(\vec{Q}_{\mathrm{R}}^{2}-\vec{Q}_{\mathrm{L}}^{2}\right)
$$

The entropy of this black hole vanishes in the supergravity approximation. However higher derivative corrections become important near the horizon since the curvature and other field strengths become large in this region. There is a general scaling argument that shows that for large $Q^{2}$ the entropy of the black hole, after taking into account the higher derivative corrections, goes as $a \sqrt{N}$ for some constant $a[10,12]$. More recently, [1-7] computed the entropy of these black holes by taking into account a specific class of higher derivative terms in the action, following earlier work on supersymmetric attractor mechanism [28-30] and the effect of higher derivative terms on the black hole entropy [20,31-38]. The higher derivative terms which were used in this calculation represent corrections to the generalized prepotential of 
the theory and are of the form $[20,39-41]$

$$
\int d^{4} x \sqrt{\operatorname{det} g}\left[\phi(S, \bar{S}) R_{\mu \nu \rho \sigma}^{-} R^{-\mu \nu \rho \sigma}+\text { c.c. }\right]+\cdots,
$$

where $g_{\mu \nu}, R_{\mu \nu \rho \sigma}^{ \pm}$, and $S$ denote, respectively, the canonical metric, the self-dual, and the anti-self-dual components of the Riemann tensor and the complex scalar field whose real and imaginary parts are given by the exponential of the dilaton field and the axion field, respectively. The function ${ }^{3}$

$$
\phi(S, \bar{S})=g(S)-\frac{K}{128 \pi^{2}} \ln (S+\bar{S})
$$

is the sum of a piece $g(S)$ that is holomorphic in $S$ and a piece proportional to $\ln (S+\bar{S})$ that is a function of both $S$ and $\bar{S}$. For large $S$, the function $g(S)$ has the form

$$
g(S)=\frac{S}{16 \pi}+\mathcal{O}\left(\mathrm{e}^{-2 \pi S}\right)
$$

Furthermore, the combination $\hat{h}(S)$, defined as

$$
\hat{h}(S) \equiv \partial_{S} g(S)+\frac{K}{32 \pi^{2}} \partial_{S} \ln \eta\left(\mathrm{e}^{-2 \pi S}\right), \quad \eta(q) \equiv q^{1 / 24} \prod_{n=1}^{\infty}\left(1-q^{n}\right),
$$

transforms as a modular form of weight two under the S-duality group of the theory [6]. The constant $K$ in equation $(2.4)$ and (2.6) is given by $[6,41]$

$$
K=24-k,
$$

and represents the effect of holomorphic anomaly [17-19]. Finally, $\cdots$ in equation (2.3) denotes various other terms which are required for the supersymmetric completion.

In the presence of the term given in equation (2.3), the black hole entropy $S_{\mathrm{BH}}$ is given by the formula $[6,20]$ :

$$
S_{\mathrm{BH}}=\frac{\pi N}{S_{0}}+64 \pi^{2} g\left(S_{0}\right)-\frac{K}{2} \ln \left(2 S_{0}\right)+C,
$$

where $S_{0}$, the value of the field $S$ at the horizon, is determined from the equation:

$$
-\frac{\pi N}{S_{0}^{2}}+64 \pi^{2} g^{\prime}\left(S_{0}\right)-\frac{K}{2 S_{0}} \simeq 0
$$

\footnotetext{
${ }^{3}$ We shall be considering those heterotic string compactifications which admit dual type II description so that we can compute $\phi(S, \bar{S})$ by working in this dual description $[6,40,41]$. A wide class of such models were constructed in $[26,27]$.
} 
and $C$ is a constant whose value is not known at present. Note that $S_{\mathrm{BH}}$ is a function only of the combination $N$ defined in equation (2.2) and does not depend on the details of the charge vector $Q$. In arriving at equations (2.8) and (2.9) one needs to take into account the correction to the Bekenstein-Hawking formula for the entropy due to higher derivative terms in the action [42-45]. It should be mentioned, however, that this formula has been derived from first principles only in the case $K=0$ where the non-holomorphic contribution to $\phi(S, \bar{S})$ is absent. It is usually difficult to supersymmetrize the non-holomorphic terms, and [20] guessed this formula for toroidally compactified heterotic string theory using the requirement of S-duality invariance. Equations (2.8) and (2.9) are generalizations of this formula for general $\mathcal{N}=4$ supersymmetric compactification [6]. The constant $C$ in equation (2.8) is not determined by the requirement of duality invariance. We should keep in mind, however, that for a given theory this is a fixed constant and is independent of the charges carried by the black hole.

We now define $\mathcal{F}_{\mathrm{BH}}(\mu)$ as the Legendre transform of the function $S_{\mathrm{BH}}(N)$ with respect to the variable $N$. Then, $S_{\mathrm{BH}}$ is the inverse Legendre transform of $\mathcal{F}(\mu)$ :

$$
S_{\mathrm{BH}}(N)=\mathcal{F}_{\mathrm{BH}}(\mu)+\mu N,
$$

with $\mu$ determined from the formula

$$
\frac{\partial \mathcal{F}_{\mathrm{BH}}}{\partial \mu}+N=0
$$

Equations (2.8) and (2.9) are identical to equations (2.10) and (2.11) provided we make the identification

$$
\begin{aligned}
\mu & =\frac{\pi}{S_{0}} \\
\mathcal{F}_{\mathrm{BH}}(\mu) & =64 \pi^{2} g\left(\frac{\pi}{\mu}\right)-\frac{K}{2} \ln \left(\frac{2 \pi}{\mu}\right)+C \\
& =\frac{4 \pi^{2}}{\mu}-\frac{24-k}{2} \ln \left(\frac{2 \pi}{\mu}\right)+C+\mathcal{O}\left(\mathrm{e}^{-2 \pi^{2} / \mu}\right) .
\end{aligned}
$$

In arriving at the second line of this equation, we have used equations (2.5) and (2.7). $\mathrm{e}^{\mathcal{F}_{\mathrm{BH}}(\mu)}$ will be called the black hole partition function.

We propose the following relation between $\mathcal{F}_{\mathrm{BH}}(\mu)$ and the degeneracy $d(Q)$ of elementary string states carrying electric charge $Q$ :

$$
d(Q) \simeq \frac{1}{2 \pi i} \mathrm{e}^{-C_{0}} \int_{\mu_{0}-i a}^{\mu_{0}+i a} \mathrm{e}^{\mathcal{F}_{\mathrm{BH}}(\mu)+\mu N}, \quad N=\frac{1}{2} Q^{2},
$$


where $\simeq$ denotes equality up to error terms which are suppressed by powers of $\mathrm{e}^{-\pi \sqrt{N}}$ relative to the leading term, $\mu_{0}$ is given by the solution of the equation

$$
\mathcal{F}_{\mathrm{BH}}^{\prime}\left(\mu_{0}\right)+N=0, \quad \operatorname{Im}\left(\mu_{0}\right)=0, \quad \operatorname{Re}\left(\mu_{0}\right)>0,
$$

$a$ is a small positive constant about which we shall say more in the next paragraph and $C_{0}$ is a constant whose value will be determined in equation (3.59). Note that according to this proposal the degeneracy of the elementary string states should be a function of the combination $N=Q^{2} / 2$ only, and should not depend on the details of the charge vector $Q$. In section 3 , we shall verify that this is true up to exponentially suppressed correction terms.

In order to understand the role of the limits of integration on the righthand side of equation (2.14), we need to analyze this integral in some detail. From equation (2.13), it follows that $\mu_{0}$ given in equation (2.17) is a saddle point of the integrand in equation (2.14) up to exponentially suppressed corrections. Up to power corrections $\mu_{0} \simeq 2 \pi / \sqrt{N}$, and the value of the integrand near this saddle point has a factor of $\mathrm{e}^{4 \pi \sqrt{N}}$, with a factor of $\mathrm{e}^{2 \pi \sqrt{N}}$ coming from the $\mathrm{e}^{\mathcal{F}_{\mathrm{BH}}\left(\mu_{0}\right)}$ term and another factor of $\mathrm{e}^{2 \pi \sqrt{N}}$ coming from the $\mathrm{e}^{\mu_{0} N}$ term. As mentioned below equation (2.6), the function $g(\pi / \mu)$ appearing in the expression (2.13) for $\mathcal{F}_{\mathrm{BH}}(\mu)$ has modular properties, and as a result there are additional divergences of the integrand near the points of the form $\mu=i \nu, \nu=2 \pi p / q$ for integer $p, q$. Near these points, $\mathcal{F}_{\mathrm{BH}}(\mu) \sim$ $4 \pi^{2} c_{p, q} /(\mu-i \nu)$ for some constant $c_{p, q}$ that depends on the fraction $p / q$ and typically is smaller for larger $q$. Since the integration contour has been chosen to have $\operatorname{Re}(\mu)=\mu_{0}$, the maximum contribution to the integrand from a singularity at $\mu=i \nu$ comes when the integration contour passes through the point $\mu=\mu_{0}+i \nu$, and at this point the integrand has a factor of order $\mathrm{e}^{2 \pi \sqrt{N}\left(1+c_{p, q}\right)+i \nu N}$. As long as $c_{p, q}$ is smaller than 1 , this contribution is exponentially suppressed compared to the contribution coming from the saddle point near $\mu_{0}$. We shall choose the constant $a$ appearing in the integration range of $\mu$ to be sufficiently small so that for all points of the form $2 \pi i p / q$ in the range $(-i a, i a)$ the coefficient $c_{p, q}$ is smaller than 1 . In this case, the dominant contribution to the integral comes from a region close to $\mu_{0}$, and hence the dependence of the integral on the constant $a$ will be exponentially suppressed as long as $a$ satisfies the criteria given above. Also in this case, we can ignore the contributions to $\mathcal{F}_{\mathrm{BH}}(\mu)$ which are suppressed by powers of $\mathrm{e}^{-\pi^{2} / \mu}$, since after integration this will produce corrections that are suppressed by powers of $\mathrm{e}^{-\pi^{2} / \mu_{0}} \sim \mathrm{e}^{-\pi \sqrt{N} / 2}$. Equations (2.13) now allows us to restate the proposals (2.14) and (2.15) as

$$
d(Q) \simeq \frac{1}{2 \pi i} \mathrm{e}^{-C_{0}} \int_{\mu_{0}-i a}^{\mu_{0}+i a} \exp \left(\mu N+\frac{4 \pi^{2}}{\mu}-\frac{24-k}{2} \ln \left(\frac{2 \pi}{\mu}\right)+C\right),
$$


where $\mu_{0}$ is the solution of the equation

$$
-\frac{4 \pi^{2}}{\mu_{0}^{2}}+\frac{24-k}{2 \mu_{0}}+N=0, \quad \mu_{0}>0 .
$$

We shall now reformulate this relation in terms of a relation between the black hole partition function $\mathrm{e}^{\mathcal{F}_{\mathrm{BH}}(\mu)}$ and the partition function of elementary string states, defined as

$$
\mathrm{e}^{\mathcal{F}(\mu)}=\frac{1}{M} \sum_{N} d_{N} \mathrm{e}^{-\mu N}
$$

where the sum over $N$ in equation (2.18) runs over all the allowed values of $N$ in the theory, and $M$ is an integer that counts the number of allowed values of $N$ per unit interval. In order to define the quantity $d_{N}$ appearing on the right-hand side of equation (2.18), we need to pick a representative charge vector $Q$ so that $Q^{2} / 2=N$ and identify $d_{N}$ as the degeneracy $d(Q)$ of states carrying charge $Q$. As mentioned earlier, the leading contribution of order $\mathrm{e}^{4 \pi \sqrt{N}}$ to $d_{N}$ is independent of the choice of the representative $Q$ that we choose, but there are corrections suppressed by powers of $\mathrm{e}^{-\pi \sqrt{N}}$ which do depend on this representative. By analyzing the sum in equation (2.18) by saddle point method, one can easily see that a contribution to $d_{N}$ that grows as $\exp (4 \pi c \sqrt{N})$ will give rise to a contribution to $\mathrm{e}^{\mathcal{F}(\mu)}$ of order $\exp \left(4 \pi^{2} c^{2} / \mu\right)$ for small $\mu$. Thus, an uncertainty in $d_{N}$ that is suppressed by powers of $\exp (-\pi \sqrt{N})$ will induce an uncertainty in the definition of $\mathcal{F}(\mu)$ that is suppressed by powers of $\mathrm{e}^{-\pi^{2} / \mu}$. This will not affect our analysis, since the proposed relation $(2.26)$ between $\mathcal{F}_{\mathrm{BH}}(\mu)$ and $\mathcal{F}(\mu)$ is expected to be valid only up to exponentially suppressed correction terms.

In order to analyze the consequence of the proposed form (2.16) for $d_{N}$ for the partition function $\mathrm{e}^{\mathcal{F}(\mu)}$, it will be convenient to work with a more general set of partition functions defined as follows. For any integer $J$, let $S_{J}$ denote the set of allowed values of $N$ in the interval $-1 \leq N<J-1$. Since there are $M$ possible values of $N$ in unit interval, the set $S_{J}$ will contain $J M$ elements. For any $\alpha \in S_{J}$, we define

$$
\mathrm{e}^{\mathcal{F}_{\alpha}^{(J)}(\mu)} \equiv J \sum_{L=0}^{\infty} d_{J L+\alpha} \mathrm{e}^{-\mu(J L+\alpha)} .
$$

As in the definition of $\mathcal{F}(\mu), \mathcal{F}_{\alpha}^{(J)}(\mu)$ also suffers from an uncertainty that is suppressed by powers of $\mathrm{e}^{-\pi^{2} / \mu}$. Using equations (2.18) and (2.19), we get

$$
\mathrm{e}^{\mathcal{F}(\mu)}=\frac{1}{J M} \sum_{\alpha \in S_{J}} \mathrm{e}^{\mathcal{F}_{\alpha}^{(J)}(\mu)} .
$$


We shall now substitute equation (2.16) into equation (2.19) to come up with a proposal for the form of $\mathcal{F}_{\alpha}^{(J)}(\mu)$ :

$$
\begin{aligned}
\mathrm{e}^{\mathcal{F}_{\alpha}^{(J)}(\mu)} \simeq & \frac{J}{2 \pi i} \mathrm{e}^{-C_{0}+C} \sum_{L=0}^{\infty} \mathrm{e}^{-\mu(J L+\alpha)} \\
& \times \int_{\mu_{0}-i a}^{\mu_{0}+i a} d \mu^{\prime} \mathrm{e}^{4 \pi^{2} / \mu^{\prime}+\mu^{\prime}(J L+\alpha)}\left(\frac{\mu^{\prime}}{2 \pi}\right)^{12-(1 / 2) k}
\end{aligned}
$$

where $\simeq$ denotes equality up to terms suppressed by powers of $\mathrm{e}^{-\pi^{2} / \mu}$ compared to the leading term. Note that in arriving at equation (2.21), we have used the proposed form of $d_{N}$ in terms of black hole entropy that is correct up to fractional errors involving powers of $\mathrm{e}^{-\pi \sqrt{N}}$, but this is okay since these induce errors in the expression for $\mathcal{F}_{\alpha}^{(J)}(\mu)$ involving powers of $\mathrm{e}^{-\pi^{2} / \mu}$. In equation (2.21), $\mu_{0}$ is the solution of equation (2.17) with $N$ replaced by $J L+\alpha$ and hence depends on $J L$. We shall now deform the $\mu^{\prime}$ integration contour so that it runs parallel to the real axis from $\mu_{0}-i a$ to a point close to the point $-i a$ on the imaginary axis, then runs till a point close to $i a$ by grazing the imaginary axis, and finally returns back to $\mu_{0}+i a$ along a line parallel to the real axis. For the first and the third component of the contour, the $\mathrm{e}^{4 \pi^{2} / \mu^{\prime}}$ part remains finite, and hence the contribution to the $\mu^{\prime}$ integral from these contours are bounded by a term of order $\mathrm{e}^{\mu_{0}(J L+\alpha)} \sim \mathrm{e}^{2 \pi \sqrt{J L+\alpha}}$. After performing the sum over $L$ for these terms, we get contributions bounded by a term of order $\mathrm{e}^{\pi^{2} / \mu}$. The middle component of the contour that grazes the imaginary $\mu^{\prime}$ axis is independent of $L$. Furthermore, for any point $\mu^{\prime}$ on this contour, $\operatorname{Re}\left(\mu-\mu^{\prime}\right)>0$ for any finite $\mu$. Hence, we can perform the sum over $L$ explicitly and write the contribution as

$$
\frac{J}{2 \pi i} \mathrm{e}^{-C_{0}+C} \mathrm{e}^{-\left(\mu-\mu^{\prime}\right) \alpha} \int_{0^{+}-i a}^{0^{+}+i a} d \mu^{\prime} \frac{1}{1-\mathrm{e}^{-J\left(\mu-\mu^{\prime}\right)}} \mathrm{e}^{4 \pi^{2} / \mu^{\prime}}\left(\frac{\mu^{\prime}}{2 \pi}\right)^{12-(1 / 2) k} .
$$

We can now evaluate the integral by deforming the $\mu^{\prime}$ integration contour to a semicircle of radius $a$ in the $\operatorname{Re}\left(\mu^{\prime}\right)>0$ region. During this process, we pick up the residue at the pole at $\mu^{\prime}=\mu$. This contribution is given by

$$
\mathrm{e}^{-C_{0}+C} \mathrm{e}^{4 \pi^{2} / \mu}\left(\frac{\mu}{2 \pi}\right)^{12-(1 / 2) k}
$$

The left-over contour integral along the semicircle $\left|\mu^{\prime}\right|=a$ does not have any exponentially large contribution, and hence this contribution is exponentially suppressed compared to equation (2.23). Furthermore, the contribution from the first and the third components of the contour, which were seen to be bounded by terms of order $\mathrm{e}^{\pi^{2} / \mu}$, are also exponentially small 
compared to equation (2.23). Thus, equation (2.23) gives the complete contribution to equation (2.21) up to exponentially suppressed terms, and we get

$$
\mathcal{F}_{\alpha}^{(J)}(\mu) \simeq \frac{4 \pi^{2}}{\mu}+\left(12-\frac{1}{2} k\right) \ln \frac{\mu}{2 \pi}+C-C_{0} \simeq \mathcal{F}_{\mathrm{BH}}(\mu)-C_{0} .
$$

Equation (2.24) implies that for small $\mu, \mathcal{F}_{\alpha}^{(J)}(\mu)$ is independent of $J$ and $\alpha$ up to correction terms involving powers of $\mathrm{e}^{-\pi^{2} / \mu}$. This information, together with equation $(2.20)$, gives

$$
\mathcal{F}_{\alpha}^{(J)}(\mu) \simeq \mathcal{F}(\mu)
$$

This in turn allows us to restate equation (2.24) $\mathrm{as}^{4}$

$$
\mathcal{F}(\mu) \simeq \mathcal{F}_{\mathrm{BH}}(\mu)-C_{0} .
$$

Equations (2.24) and (2.26) are different ways of restating the proposed relation (2.14) between black hole entropy and degeneracy of elementary string states. By taking the inverse Legendre transform of both sides of equation (2.26), we may state the proposal in yet another form:

$$
\widetilde{S}_{\text {stat }}(N) \simeq S_{\mathrm{BH}}(N)-C_{0},
$$

where $\widetilde{S}_{\text {stat }}(N)$ is the inverse Legendre transform of $\mathcal{F}(\mu)$ :

$$
\widetilde{S}_{\text {stat }}(N)=\mathcal{F}(\mu)+\mu N, \quad \frac{\partial \mathcal{F}(\mu)}{\partial \mu}+N=0 .
$$

$\simeq$ in equation $(2.27)$ represents equality up to error terms involving powers of $\mathrm{e}^{-\pi \sqrt{N}}$.

\section{Counting degeneracy of BPS states in CHL compactification}

In this section, we shall generalize the analysis of $[6,7]$ to compute the degeneracy of half-BPS elementary string states in a general class of CHL models [22-27] and verify its proposed relation to the black hole entropy as given in equations (2.14) and (2.16). These models are obtained by beginning with heterotic string compactified on $T^{6}$ and then modding out the theory by the action of an abelian group $G$. In particular, [26, 27] constructed a

\footnotetext{
${ }^{4}$ This is the way the conjecture was stated earlier in $[3,6]$. There we worked with a special class of theories where the compactification manifold has an $S^{1}$ factor and chose the representative $d_{N}$ in equation (2.19) from a special class of elementary string states which carry $w$ units of winding charge and $n$ units of momentum along this circle without carrying any other charges. For these states, $Q^{2}=2 n w$, and one can check explicitly that $d_{N}$ depends only on the combination $n w$ and not individually on $n$ and $w$.
} 
wide class of such models which admit dual type II description by beginning with heterotic string compactification on a six-dimensional torus of the form $T^{4} \times S^{1} \times \widetilde{S}^{1}$, and then modding out the theory by a $Z_{m} \times Z_{n}$ group, where $Z_{m}\left(Z_{n}\right)$ acts on $S^{1}\left(\widetilde{S}^{1}\right)$ by a shift of order $m(n)$ and also acts as a $Z_{m}\left(Z_{n}\right)$ symmetry transformation on the rest of the conformal field theory involving the coordinates of $T^{4}$ and the other 16 left-moving world-sheet scalars associated with $E_{8} \times E_{8}$ gauge group. The action of a typical element $g$ of the orbifold group can be regarded as a combination of a shift $a_{g}$ and a rotation $R_{g}$ on the signature $(22,6)$ Narain lattice $\Gamma$ associated with the toroidal compactification $[46,47]$ :

$$
P \rightarrow R_{g} P+a_{g}, \quad P \in \Gamma .
$$

The rotation part also acts on the oscillators. The set of $R_{g}$ 's for $g \in G$ form a group that describes the rotational part of $G$. We shall call this group $R_{G}$. In order to preserve $\mathcal{N}=4$ supersymmetry, we need to ensure that $R_{G}$ acts trivially on the right-handed world-sheet fields. If we assume that the full group $R_{G}$ leaves $(22-k)$ of the 22 left-moving directions invariant, then for any given $g, R_{g}$ can be characterized by $k / 2$ rotation angles $2 \pi \phi_{1}(g), \ldots, 2 \pi \phi_{k / 2}(g) .^{5} \quad$ We shall denote by $X^{j}\left(1 \leq j \leq \frac{k}{2}\right)$ the complex world-sheet scalars labelling the planes of rotation so that the effect of rotation on the corresponding left-handed oscillators $\alpha_{-n}^{j}$ is represented by

$$
\alpha_{-n}^{j} \rightarrow \mathrm{e}^{2 \pi i \phi_{j}(g)} \alpha_{-n}^{j}
$$

The corresponding complex conjugate oscillator transforms with a phase $\mathrm{e}^{-2 \pi i \phi_{j}(g)}$. Thus, without any loss of generality, we can restrict $\phi_{j}(g)$ 's to be in the range

$$
0 \leq \phi_{j}(g) \leq \frac{1}{2}
$$

For future reference, it will be useful to set up some notations and definitions at this stage. Let $V$ denote the $(22+6)$-dimensional vector space in which the lattice $\Gamma$ is embedded. For a given group element $g$, we denote by $V_{\perp}(g)$ the subspace of $V$ that is left invariant by $R_{g}$, and by $V_{\|}(g)$ its orthogonal subspace. Thus, the planes of rotation associated with a given group element $g$ are orthogonal to $V_{\perp}(g)$ and lie along $V_{\|}(g)$. We also define

$$
V_{\perp}=\bigcap_{g \in G} V_{\perp}(g), \quad V_{\|}=\bigcup_{g \in G} V_{\|}(g) .
$$

In others words, $V_{\perp}$ is the subspace of $V$ that is left invariant by the entire group $G$, and $V_{\|}$is its orthogonal subspace. The total dimension of $V_{\perp}$ is $(28-k)$. This is the number of $\mathrm{U}(1)$ gauge fields in the resulting theory.

\footnotetext{
${ }^{5}$ For a particular element $g$, some of the $\phi_{j}(g)$ 's may vanish, but for each $j, \phi_{j}(g) \neq 0$ for at least one $g \in G$.
} 
Finally, let us define

$$
\Lambda_{\perp}(g)=\Gamma \bigcap V_{\perp}(g), \quad \Lambda_{\perp}=\Gamma \bigcap V_{\perp}=\bigcap_{g} \Lambda_{\perp}(g),
$$

and

$$
\Lambda_{\|}(g)=\Gamma \bigcap V_{\|}(g), \quad \Lambda_{\|}=\Gamma \bigcap V_{\|}
$$

Let us now begin analyzing the spectrum of half-BPS elementary string states. We first consider untwisted sector states. Before the orbifold projection, a generic BPS state is obtained by keeping the right-moving sector of the state at the lowest $L_{0}$ eigenvalue allowed by GSO projection and considering arbitrary excitations on the left-moving sector. If we denote by $\left(\vec{P}_{\mathrm{L}}, \vec{P}_{\mathrm{R}}\right)$ the left and right components of the momentum vector and by $N_{\mathrm{L}}$ the total level of left-handed oscillator excitations, then the level-matching condition gives

$$
N_{\mathrm{L}}-1+\frac{1}{2}\left(\vec{P}_{\mathrm{L}}^{2}-\vec{P}_{\mathrm{R}}^{2}\right)=0 .
$$

While in the original theory, all the components of $P$ act as sources for electric fields, in the orbifold theory, only the components of $P$ along $V_{\perp}$ act as sources for electric fields. We shall denote by $Q=\left(\vec{Q}_{\mathrm{L}}, \vec{Q}_{\mathrm{R}}\right)$ the projection of $P$ along $V_{\perp}$ and by $P_{\|}=\left(\vec{P}_{\| \mathrm{L}}, 0\right)$ the projection of $P$ along $V_{\|}$. The requirement of $\mathcal{N}=4$ supersymmetry forces $V_{\|}$to be directed fully along the left-handed component of the lattice. As a result, $P_{\|}$has no righthanded component and $\vec{P}_{\mathrm{R}}=\vec{Q}_{\mathrm{R}}$. Equation (3.7) may now be rewritten as:

$$
N_{L}-1+\frac{1}{2} \vec{P}_{\| \mathrm{L}}^{2}=N
$$

where

$$
N \equiv \frac{1}{2}\left(\vec{Q}_{\mathrm{R}}^{2}-\vec{Q}_{\mathrm{L}}^{2}\right)
$$

If two vectors $P$ and $P^{\prime}$ in $\Lambda$ correspond to the same charge vector $Q$, then $P-P^{\prime}=\left(\vec{P}_{\| \mathrm{L}}-\vec{P}_{\| \mathrm{L}}^{\prime}, 0\right)$. Thus, $\vec{P}_{\| \mathrm{L}}-\vec{P}_{\| \mathrm{L}}^{\prime} \in \Lambda_{\|}$. This shows that, for a given $Q$, the allowed values of $\vec{P}_{\| \mathrm{L}}$ are of the form

$$
\vec{P}_{\| \mathrm{L}}=\vec{K}(Q)+\vec{p}, \quad \vec{p} \in \Lambda_{\|},
$$

where $\vec{K}(Q)$ is a fixed vector in $V_{\|}$lying within the unit cell of $\Lambda_{\|}$.

We want to count the number of $G$ invariant BPS states $d(Q)$ for a fixed $Q$. This is best done by taking the trace of $\sum_{g \in G} g$ over all states carrying the given electric charge $Q$ and then dividing the answer by the total number 
of elements $n_{G}$ of the group. Clearly, the contribution to $\operatorname{Tr}(g)$ will come from only those $\vec{P}_{\| \mathrm{L}}$ which are invariant under $R_{g}$. This requires

$$
\vec{p}+\vec{K}(Q) \in V_{\perp}(g) .
$$

Acting on the Fock vacuum carrying such a momentum $P$, the group element $g$ produces a phase

$$
\mathrm{e}^{2 \pi i a_{g} \cdot P}=\mathrm{e}^{2 \pi i a_{g} \cdot Q} \mathrm{e}^{-2 \pi i \vec{a}_{g \mathrm{~L}} \cdot(\vec{p}+\vec{K}(Q))} .
$$

If we denote by $d^{(\mathrm{osc})}\left(N_{\mathrm{L}}, g\right)$ the number of ways we can construct total oscillator level $N_{\mathrm{L}}$ out of the 24 left-moving oscillators, weighted by the action of the group element $g$ on that combination, then we may express the total number of BPS states carrying charge $Q$ as

$$
\begin{aligned}
d(Q)= & \frac{16}{n_{G}} \sum_{g \in G} \sum_{N_{\mathrm{L}}=0}^{\infty} d^{(\mathrm{osc})}\left(N_{\mathrm{L}}, g\right) \mathrm{e}^{2 \pi i a_{g} \cdot Q} \mathrm{e}^{-2 \pi i \vec{a}_{g \mathrm{~L}} \cdot \vec{K}(Q)} \\
& \times \sum_{\substack{\vec{p} \in \Lambda_{\|} \\
\vec{p}+\vec{K}(Q) \in V_{\perp}(g)}} \mathrm{e}^{-2 \pi i \vec{a}_{g \mathrm{~L}} \cdot \vec{p}} \delta_{N_{\mathrm{L}}-1+(1 / 2)(\vec{p}+\vec{K}(Q))^{2}, N} \cdot
\end{aligned}
$$

The factor of 16 in this equation counts the number of states in a single BPS supermultiplet.

In a sector twisted by a group element $g^{\prime}$, the oscillators of $X^{j}$ are fractionally moded with modes $\alpha_{-n+\phi_{j}\left(g^{\prime}\right)}^{j}$. In this case, the momenta $P$ carried by the state lie in the vector space $V_{\perp}\left(g^{\prime}\right)$ and are of the form [48]

$$
P=\widetilde{a}_{g^{\prime}}+\lambda, \quad \lambda \in \Lambda_{\perp}\left(g^{\prime}\right)^{*},
$$

where $\widetilde{a}_{g^{\prime}}$ is the projection of the vector $a_{g^{\prime}}$ into $V_{\perp}\left(g^{\prime}\right)$, and $\Lambda_{\perp}\left(g^{\prime}\right)^{*}$ is the lattice dual to $\Lambda_{\perp}\left(g^{\prime}\right)$. If we denote by $N_{L}$ the total level of the left-handed oscillators and by $\left(\vec{P}_{\mathrm{L}}, \vec{P}_{\mathrm{R}}\right)$ the left and the right-handed components of the charge vector as before, the level-matching condition reads

$$
N_{\mathrm{L}}-1+\frac{1}{2}\left(\vec{P}_{\mathrm{L}}^{2}-\vec{P}_{\mathrm{R}}^{2}\right)+\frac{1}{2} \sum_{j=1}^{k / 2} \phi_{j}\left(g^{\prime}\right)\left(1-\phi_{j}\left(g^{\prime}\right)\right)=0,
$$

where the last term on the left-hand side of this equation accounts for the $\bar{L}_{0}$ eigenvalue of the ground state of the twisted sector. As before, the electric charge vector $Q=\left(\vec{Q}_{\mathrm{L}}, \vec{Q}_{\mathrm{R}}\right)$ is given by the projection of $P$ into $V_{\perp}$. For a generic CHL compactification of the type described in [26, 27], all states carrying a given charge $Q$ arises from a single twisted sector since the projection of $a_{g^{\prime}}$ into $V_{\perp}$ are different for different $g^{\prime}$. We shall denote by $g_{Q}^{\prime}$ the twist associated with the charge vector $Q$. We also denote by $P_{\|}=\left(\vec{P}_{\| \mathrm{L}}, 0\right)$ the projection of $P$ into $V_{\|}$. Then using equation (3.14) and 
an argument identical to the one that led to equation (3.10), we can show that $\vec{P}_{\| \mathrm{L}}$ must have the form:

$$
\vec{P}_{\| \mathrm{L}}=\vec{K}(Q)+\vec{p}, \quad \vec{p} \in \Lambda_{1}\left(g_{Q}^{\prime}\right),
$$

where

$$
\Lambda_{1}\left(g^{\prime}\right)=\Lambda_{\perp}\left(g^{\prime}\right)^{*} \bigcap V_{\|},
$$

and $\vec{K}(Q)$ is some vector in $V_{\|} \cap V_{\perp}\left(g_{Q}^{\prime}\right)$ within the unit cell of the lattice $\Lambda_{\perp}\left(g_{Q}^{\prime}\right)^{*} \cap V_{\|}$. Equation (3.15) now takes the form:

$$
\begin{aligned}
N_{L}- & +\frac{1}{2}(\vec{p}+\vec{K}(Q))^{2} \\
& +\frac{1}{2} \sum_{j=1}^{k / 2} \phi_{j}\left(g_{Q}^{\prime}\right)\left(1-\phi_{j}\left(g_{Q}^{\prime}\right)\right)=N, \quad N \equiv \frac{1}{2}\left(\vec{Q}_{R}^{2}-\vec{Q}_{L}^{2}\right) .
\end{aligned}
$$

As in the case of untwisted sector states, we want to count the number of $G$ invariant BPS states $d(Q)$ for a fixed $Q$ in a sector twisted by some fixed group element $g_{Q}^{\prime}$. Again this is done by taking the trace of $\sum_{g \in G} g$ over all states carrying the given electric charge $Q$ and then dividing the answer by the total number of elements $n_{G}$ of the group. Non-vanishing contribution to the trace will come from only those $\vec{P}_{\| \mathrm{L}}$ which are invariant under $R_{g}$. Thus, we must have

$$
\vec{p}+\vec{K}(Q) \in V_{\perp}(g) .
$$

Let us denote by $d^{(\mathrm{vac})}\left(g^{\prime}\right)$ the degeneracy of the ground state in the sector twisted by $g^{\prime}$ - this is the generalization of the number of fixed points for a symmetric orbifold. We shall label these vacua by the index $r$ running from 1 to $d^{(\mathrm{vac})}\left(g^{\prime}\right)$. Acting on the $r$ th Fock vacuum carrying momentum $P$, the group element $g$ produces a phase

$$
\mathrm{e}^{i \chi_{r}\left(g_{Q}^{\prime}, g\right)} \mathrm{e}^{2 \pi i a_{g} \cdot P}
$$

where we have allowed for the possibility that acting on the $r$ th vacuum in the sector twisted by $g^{\prime}$, the action of the group element $g$ may produce a momentum independent phase $\mathrm{e}^{i \chi_{r}\left(g^{\prime}, g\right)}$. We note, however, that if $g$ is the identity element, then this phase must be trivial:

$$
\mathrm{e}^{i \chi_{r}\left(g^{\prime}, 1\right)}=1 .
$$

If we now denote by $d^{\text {(osc) }}\left(N_{L}, g^{\prime}, g\right)$ the number of ways we can construct total oscillator level $N_{L}$ out of the 24 left-moving oscillators in the sector 
twisted by $g^{\prime}$, weighted by the action of the group element $g$ on that combination, and then we may write

$$
\begin{aligned}
d(Q)= & \frac{16}{n_{G}} \sum_{r=1}^{d^{(\mathrm{vac})}\left(g_{Q}^{\prime}\right)} \sum_{g \in G} \mathrm{e}^{i \chi_{r}\left(g_{Q}^{\prime}, g\right)} \sum_{N_{\mathrm{L}}=0}^{\infty} d^{(\mathrm{osc})}\left(N_{\mathrm{L}}, g_{Q}^{\prime}, g\right) \mathrm{e}^{2 \pi i a_{g} \cdot Q} \mathrm{e}^{-2 \pi i \vec{a}_{g \mathrm{~L}} \cdot \vec{K}(Q)} \\
& \times \sum_{\substack{\vec{p} \in \Lambda_{1}\left(g_{Q}^{\prime}\right) \\
\vec{p}+\vec{K}(Q) \in V_{\perp}(g)}} \mathrm{e}^{-2 \pi i \vec{a}_{g \mathrm{~L}} \cdot \vec{p}} \delta_{N_{\mathrm{L}}-1+(1 / 2)(\vec{p}+\vec{K}(Q))^{2}+(1 / 2) \sum_{j=1}^{k / 2} \phi_{j}\left(g_{Q}^{\prime}\right)\left(1-\phi_{j}\left(g_{Q}^{\prime}\right)\right), N},
\end{aligned}
$$

where we have used the fact that for a momentum of the type given in equation (3.16),

$$
a_{g} \cdot P=a_{g} \cdot Q-a_{g \mathrm{~L}} \cdot(\vec{p}+\vec{K}(Q)) .
$$

Note that equation (3.13) can be regarded as a special case of equation (3.22) by setting $g_{Q}^{\prime}=1$ in the latter equation. Thus, once we compute $d(Q)$ using equation (3.22), we do not need to compute equation (3.13) separately.

In order to evaluate the right-hand side of equation (3.22), we shall rewrite this equation in a slightly different way. We write

$$
d(Q)=F(Q, N), \quad N \equiv \frac{1}{2}\left(\vec{Q}_{\mathrm{L}}^{2}-\vec{Q}_{\mathrm{R}}^{2}\right),
$$

where

$$
\begin{aligned}
& F(Q, \widehat{N}) \\
& \equiv \frac{16}{n_{G}} \sum_{r=1}^{d^{(\mathrm{vac})}\left(g_{Q}^{\prime}\right)} \sum_{g \in G} \mathrm{e}^{i \chi_{r}\left(g_{Q}^{\prime}, g\right)} \sum_{N_{L}=0}^{\infty} d^{(\mathrm{osc})}\left(N_{\mathrm{L}}, g_{Q}^{\prime}, g\right) \mathrm{e}^{2 \pi i a_{g} \cdot Q} \mathrm{e}^{-2 \pi i \vec{a}_{g \mathrm{~L}} \cdot \vec{K}(Q)} \\
& \quad \times \sum_{\substack{\vec{p} \in \Lambda_{1}\left(g_{Q}^{\prime}\right) \\
\vec{p}+\vec{K}(Q) \in V_{\perp}(g)}} \mathrm{e}^{-2 \pi i \vec{a}_{g \mathrm{~L}} \cdot \vec{p}} \delta_{N_{\mathrm{L}}-1+(1 / 2)(\vec{p}+\vec{K}(Q))^{2}+(1 / 2) \sum_{j=1}^{k / 2} \phi_{j}\left(g_{Q}^{\prime}\right)\left(1-\phi_{j}\left(g_{Q}^{\prime}\right)\right), \widehat{N}}
\end{aligned}
$$

Note that in equation (3.25), we have regarded $\widehat{N}$ as an independent variable not related to $Q$. This allows us to introduce a "partition function:"

$$
\widetilde{F}(Q, \mu)=\sum_{\widehat{N}} F(Q, \widehat{N}) \mathrm{e}^{-\mu \widehat{N}}
$$


where the sum over $\widehat{N}$ runs over all values for which $F(Q, \widehat{N})$ is non-zero. By the left-right level-matching condition, these are of the form ${ }^{6}$

$$
\widehat{N}=N_{0}+f(Q),
$$

where $N_{0}$ is an integer and $f(Q)$ is a fixed number between 0 and 1 which measures the fractional part of $N \equiv \frac{1}{2} Q^{2}$. Thus, we also have the reverse relation:

$$
F(Q, \widehat{N})=\frac{1}{2 \pi i} \int_{\epsilon-i \pi}^{\epsilon+i \pi} \widetilde{F}(Q, \mu) \mathrm{e}^{\mu \widehat{N}}
$$

where $\epsilon$ is any real positive number. We shall first compute $\widetilde{F}(Q, \mu)$, and then use equation $(3.28)$ to compute $F(Q, \widehat{N})$.

Using equations (3.25) and (3.26), we get

$$
\begin{aligned}
& \widetilde{F}(Q, \mu) \\
& =\frac{16}{n_{G}} \exp \left(\mu\left(1-\frac{1}{2} \sum_{j=1}^{k / 2} \phi_{j}\left(g_{Q}^{\prime}\right)\left(1-\phi_{j}\left(g_{Q}^{\prime}\right)\right)\right)\right) \\
& \quad \sum_{r=1}^{d^{(\mathrm{vac})}\left(g_{Q}^{\prime}\right)} \sum_{g \in G} \mathrm{e}^{i \chi_{r}\left(g_{Q}^{\prime}, g\right)} \mathrm{e}^{2 \pi i a_{g} \cdot Q} \mathrm{e}^{-2 \pi i \vec{a}_{g \mathrm{~L}} \cdot \vec{K}(Q)} \widetilde{F}^{(\mathrm{osc})}\left(g_{Q}^{\prime}, g, \mu\right) \widetilde{F}^{(\mathrm{lat})}(Q, g, \mu),
\end{aligned}
$$

where

$$
\widetilde{F}^{\text {(osc) }}\left(g^{\prime}, g, \mu\right)=\sum_{N_{L}=0}^{\infty} d^{(\text {osc })}\left(N_{L}, g^{\prime}, g\right) \mathrm{e}^{-\mu N_{L}}
$$

and

$$
\widetilde{F}^{(\text {lat })}(Q, g, \mu)=\sum_{\substack{\vec{p} \in \Lambda_{1}\left(g_{Q}^{\prime}\right) \\ \vec{p}+\vec{K}(Q) \in V_{\perp}(g)}} \mathrm{e}^{-2 \pi i \vec{a}_{g \mathrm{~L}} \cdot \vec{p}} \exp \left(-\frac{1}{2} \mu(\vec{p}+\vec{K}(Q))^{2}\right) .
$$

In order to compute $\widetilde{F}^{\text {(osc) }}\left(g^{\prime}, g, \mu\right)$, we note that under the action of the group element $g$, the oscillator $\alpha_{-n+\phi_{j}\left(g^{\prime}\right)}^{j}$ picks up a phase of $\mathrm{e}^{2 \pi i \phi_{j}(g)}$. For a given group element $g^{\prime}$, let us denote by the $A\left(g^{\prime}\right)$ the subset of the $k / 2$

${ }^{6}$ This follows from the fact that $F(Q, \widehat{N})$ counts the number of states in the conformal field theory which carry charge $Q$, have their right-handed oscillator excitations at the minimal level allowed by GSO projection, and have $\left(\bar{L}_{0}-L_{0}\right)$ eigenvalue $\widehat{N}-\frac{1}{2} Q^{2}$. Since the requirement of one loop modular invariance forces all states in the CFT to carry integer $\bar{L}_{0}-L_{0}$ eigenvalue, $\widehat{N}-\frac{1}{2} Q^{2}$ must be an integer. Note, however, that individual terms in the sum in equation (3.25) do not satisfy the $\widehat{N}-\frac{1}{2} Q^{2}=$ integer condition. Only after summing over $g$, which corresponds to projecting onto $G$ invariant states, the unwanted terms cancel. 
indices $j$ for which the $\phi_{j}\left(g^{\prime}\right)$ are non-zero and by $B\left(g^{\prime}\right)$ the set complementary to $A\left(g^{\prime}\right)$ in the set $(1,2, \ldots, k / 2){ }^{7}$ In this case,

$$
\operatorname{order}\left(A_{g^{\prime}}\right)=\frac{1}{2} \operatorname{dim} V_{\|}\left(g^{\prime}\right) \equiv \frac{1}{2} k_{g^{\prime}}, \quad \operatorname{order}\left(B_{g^{\prime}}\right)=\frac{1}{2}\left(k-k_{g^{\prime}}\right) .
$$

Let us define

$$
\tau=\frac{i \mu}{2 \pi}, \quad q=\mathrm{e}^{-\mu}=\mathrm{e}^{2 \pi i \tau} .
$$

Then, $\widetilde{F}^{(\text {osc })}\left(g^{\prime}, g, \mu\right)$ is given by

$$
\begin{aligned}
\widetilde{F}^{\text {(osc) }}\left(g^{\prime}, g, \mu\right) \\
=\left(\prod_{n=1}^{\infty} \frac{1}{1-q^{n}}\right)^{24-k} \prod_{j \in B\left(g^{\prime}\right)}\left(\prod_{n=1}^{\infty} \frac{1}{1-\mathrm{e}^{2 \pi i \phi_{j}(g)} q^{n}} \frac{1}{1-\mathrm{e}^{-2 \pi i \phi_{j}(g)} q^{n}}\right) \\
\quad \times \prod_{j \in A\left(g^{\prime}\right)}\left(\prod_{n=1}^{\infty} \frac{1}{1-\mathrm{e}^{2 \pi i \phi_{j}(g)} q^{n-\phi_{j}\left(g^{\prime}\right)}} \prod_{n=0}^{\infty} \frac{1}{1-\mathrm{e}^{-2 \pi i \phi_{j}(g)} q^{n+\phi_{j}\left(g^{\prime}\right)}}\right) .
\end{aligned}
$$

Using the Jacobi $\vartheta$ function

$$
\vartheta_{1}(z \mid \tau)=2 q^{1 / 12} \eta(q) \sin (\pi z) \prod_{n=1}^{\infty}\left(1-q^{n} \mathrm{e}^{2 \pi i z}\right)\left(1-q^{n} \mathrm{e}^{-2 \pi i z}\right)
$$

where

$$
\eta(q)=q^{1 / 24} \prod_{n=1}^{\infty}\left(1-q^{n}\right)
$$

we can rewrite equation (3.34) as

$$
\begin{aligned}
\widetilde{F}^{(\mathrm{osc})}\left(g^{\prime}, g, \mu\right)= & q(\eta(q))^{3 k / 2-24} \prod_{j \in B\left(g^{\prime}\right)} \frac{2 \sin \left(\pi \phi_{j}(g)\right)}{\vartheta_{1}\left(\phi_{j}(g) \mid \tau\right)} \\
& \times \prod_{j \in A\left(g^{\prime}\right)} \frac{\mathrm{e}^{i \pi\left(\phi_{j}(g)-\tau \phi_{j}\left(g^{\prime}\right)\right)}}{i \vartheta_{1}\left(\phi_{j}(g)-\tau \phi_{j}\left(g^{\prime}\right) \mid \tau\right)} .
\end{aligned}
$$

If some of the $\phi_{j}(g)$ s for $j \in B_{g^{\prime}}$ vanish, then we should replace the corresponding term in the product by its limit as $\phi_{j}(g) \rightarrow 0$ :

$$
\lim _{\phi_{j}(g) \rightarrow 0} \frac{2 \sin \left(\pi \phi_{j}(g)\right)}{\vartheta_{1}\left(\phi_{j}(g) \mid \tau\right)}=(\eta(q))^{-3} .
$$

\footnotetext{
${ }^{7}$ Thus, the $x^{j}$ s for $j \in A\left(g^{\prime}\right)$ and their complex conjugate coordinates span the vector space $V_{\|}\left(g^{\prime}\right)$.
} 
We are interested in the behaviour of $\widetilde{F}^{(\text {osc })}\left(g^{\prime}, g, \mu\right)$ for small $\mu$, i.e., for small $\tau$, since we shall see later that up to exponentially suppressed corrections, the contribution to equation (3.28) comes from a small region around the origin. Using the modular transformation properties of $\eta(\tau)$ and $\vartheta_{1}(z \mid \tau)$, it can be seen that, in this limit, if $z$ remains fixed with $0 \leq \operatorname{Re}(z) \leq \frac{1}{2}$,

$$
\begin{array}{r}
\eta(q) \simeq \mathrm{e}^{-\pi^{2} / 6 \mu} \sqrt{\frac{2 \pi}{\mu}}, \\
\vartheta_{1}(z \mid \tau) \simeq \mathrm{e}^{-\pi^{2} / 2 \mu} \mathrm{e}^{2 \pi^{2} z(1-z) / \mu} \sqrt{\frac{2 \pi}{\mu}}
\end{array}
$$

where $\simeq$ denotes equality up to terms which are suppressed by powers of $\mathrm{e}^{-\pi^{2} / \mu}$. Using equations (3.37)-(3.40), we see that in the $\mu \rightarrow 0$ limit the ratio

$$
\frac{\widetilde{F}^{\text {(osc })}\left(g^{\prime}, g, \mu\right)}{\widetilde{F}^{\text {(osc) }}\left(g^{\prime}, 1, \mu\right)}
$$

is exponentially small for any $g \neq 1$ due to the non-vanishing $\phi_{j}(g)$ 's. As a result, $\widetilde{F}^{(\mathrm{osc})}\left(g_{Q}^{\prime}, 1, \mu\right)$, for which all the $\phi_{j}(g)$ 's vanish, is exponentially large compared to all other $\widetilde{F}^{\text {(osc) }}\left(g_{Q}^{\prime}, g, \mu\right)$ appearing in equation (3.29). It is easy to see that the $\widetilde{F}^{(\text {lat })}(Q, g, \mu)$ factor cannot compensate for this suppression-indeed, from equation $(3.31)$, it follows that $\widetilde{F}^{(\text {lat })}(Q, 1, \mu)$ is greater than or equal to $\widetilde{F}^{\text {(lat) }}(Q, g, \mu)$ for any $g$. Thus, the sum over $g$ in equation (3.29) can be replaced by a single term corresponding to $g=1$ if we are willing to ignore corrections involving powers of $\mathrm{e}^{-\pi^{2} / \mu}$.

This shows that in order to compute $\widetilde{F}(Q, \mu)$ up to exponentially suppressed contributions, we only need to evaluate $\widetilde{F}^{(\text {osc })}\left(g_{Q}^{\prime}, 1, \mu\right)$ and $\widetilde{F}^{\text {(lat) }}$ $(Q, 1, \mu)$. This simplifies the analysis enormously since all the $\phi_{j}(g)$ 's vanish in equation (3.37), and hence all the terms in the set $B_{g^{\prime}}$ are now replaced by the right hand side of equation (3.38). Using equation (3.32) this gives

$$
\widetilde{F}^{\text {(osc) }}\left(g^{\prime}, 1, \mu\right)=q(\eta(q))^{(3 / 2) k_{g^{\prime}}-24} \prod_{j \in A\left(g^{\prime}\right)} \frac{\mathrm{e}^{-i \pi \tau \phi_{j}\left(g^{\prime}\right)}}{i \vartheta_{1}\left(-\tau \phi_{j}\left(g^{\prime}\right) \mid \tau\right)}
$$

In the $\tau \rightarrow 0$ limit,

$$
\vartheta_{1}\left(-\tau \phi_{j}\left(g^{\prime}\right) \mid \tau\right) \simeq-i \sqrt{\frac{2 \pi}{\mu}} \mathrm{e}^{-\pi^{2} / 2 \mu} \mathrm{e}^{(\mu / 2) \phi_{j}\left(g^{\prime}\right)^{2}} 2 \sin \left(\pi \phi_{j}\left(g^{\prime}\right)\right)
$$


up to exponentially suppressed terms. Equations (3.39), (3.42), and (3.43) now give

$$
\begin{aligned}
\widetilde{F}^{\text {(osc })}\left(g^{\prime}, 1, \mu\right) \simeq & \mathrm{e}^{4 \pi^{2} / \mu} \exp \left(-\mu\left(1-\frac{1}{2} \sum_{j=1}^{k / 2} \phi_{j}\left(g^{\prime}\right)\left(1-\phi_{j}\left(g^{\prime}\right)\right)\right)\right) \\
& \times\left(\frac{\mu}{2 \pi}\right)^{12-(1 / 2) k_{g^{\prime}}} \prod_{j \in A_{g^{\prime}}} \frac{1}{2 \sin \left(\pi \phi_{j}\left(g^{\prime}\right)\right)} .
\end{aligned}
$$

In writing the argument of the exponential in equation (3.44), we have replaced the sum over $j \in A_{g^{\prime}}$ by the sum over all values of $j$ in the range $(1, k / 2)$, since $\phi_{j}\left(g^{\prime}\right)$ vanishes outside the set $A_{g^{\prime}}$ anyway.

Let us now turn to the analysis of $\widetilde{F}^{\text {(lat) }}(Q, 1, \mu)$. In this case, $V_{\perp}(g=$ $1)=V$ and the condition $\vec{p}+\vec{K}(Q) \in V_{\perp}(g)$ is trivially satisfied. Thus, equation (3.31) simplifies to:

$$
\widetilde{F}^{(\text {lat })}(Q, 1, \mu)=\sum_{\vec{p} \in \Lambda_{1}\left(g_{Q}^{\prime}\right)} \exp \left(-\frac{1}{2} \mu(\vec{p}+\vec{K}(Q))^{2}\right) .
$$

The dimension of the lattice $\Lambda_{1}\left(g^{\prime}\right)$ defined in equation (3.17) is that of $V_{\perp}\left(g^{\prime}\right) \bigcap V_{\|}$. This counts the number of directions in the $k$-dimensional vector space $V_{\|}$which is left invariant under the element $g^{\prime}$. Comparing this with equation (3.32), we see that

$$
\operatorname{dim} \Lambda_{1}\left(g^{\prime}\right)=k-k_{g^{\prime}} .
$$

With the help of equation (3.46) and Poisson resummation, we may re-express equation $(3.45)$ as

$$
\begin{aligned}
\widetilde{F}^{\text {(lat })}(Q, 1, \mu)= & \frac{1}{v_{\Lambda_{1}\left(g_{Q}^{\prime}\right)}}\left(\frac{\mu}{2 \pi}\right)^{(1 / 2)\left(k_{g_{Q}^{\prime}}-k\right)} \\
& \times \sum_{\vec{q} \in \Lambda_{1}\left(g_{Q}^{\prime}\right)^{*}} \exp \left(-\frac{2 \pi^{2}}{\mu} \vec{q}^{2}+2 \pi i \vec{q} \cdot \vec{K}(Q)\right),
\end{aligned}
$$

where for any lattice $\Lambda, v_{\Lambda}$ denotes the volume of the unit cell of the lattice $\Lambda$ and $\Lambda^{*}$ denotes its dual lattice. Thus, up to exponentially suppressed contribution, we have

$$
\widetilde{F}^{(\text {lat })}(Q, 1, \mu) \simeq \frac{1}{v_{\Lambda_{1}\left(g_{Q}^{\prime}\right)}}\left(\frac{\mu}{2 \pi}\right)^{(1 / 2)\left(k_{g_{Q}^{\prime}}-k\right)} .
$$


Finally, we need the value of $d^{(\mathrm{vac})}\left(g^{\prime}\right)$ - the degeneracy of the ground state of the sector twisted by $g^{\prime}$. This was computed in [48] and is given by

$$
d^{(\mathrm{vac})}\left(g^{\prime}\right)=\frac{1}{v_{\Lambda_{\perp}\left(g^{\prime}\right)}} \prod_{j \in A\left(g^{\prime}\right)}\left(2 \sin \left(\pi \phi_{j}\left(g^{\prime}\right)\right) .\right.
$$

We are now ready to compute $\widetilde{F}(Q, \mu)$. Restricting the sum over $g$ in equation (3.29) to only over the identity element, and using equations (3.21), (3.44), (3.48), and (3.49), we get

$$
\begin{aligned}
\widetilde{F}(Q, \mu) \simeq & 16 \frac{d^{(\mathrm{vac})}\left(g_{Q}^{\prime}\right)}{n_{G}} \exp \left(\mu\left(1-\frac{1}{2} \sum_{j=1}^{k / 2} \phi_{j}\left(g_{Q}^{\prime}\right)\left(1-\phi_{j}\left(g_{Q}^{\prime}\right)\right)\right)\right) \\
& \times \widetilde{F}^{(\mathrm{osc})}\left(g_{Q}^{\prime}, 1, \mu\right) \widetilde{F}^{(\mathrm{lat})}(Q, 1, \mu) \\
\simeq & \frac{16}{n_{G} v_{\Lambda_{1}\left(g_{Q}^{\prime}\right)} v_{\Lambda_{\perp}\left(g_{Q}^{\prime}\right)}} \mathrm{e}^{4 \pi^{2} / \mu}\left(\frac{\mu}{2 \pi}\right)^{12-(1 / 2) k} .
\end{aligned}
$$

From definition (3.4), it follows that $V_{\perp}\left(g^{\prime}\right)$ has an orthogonal decomposition:

$$
V_{\perp}\left(g^{\prime}\right)=V_{\perp} \oplus\left(V_{\perp}\left(g^{\prime}\right) \bigcap V_{\|}\right) .
$$

Then, given any lattice $\Lambda \in V_{\perp}\left(g^{\prime}\right)$, we have [49]

$$
v_{\Lambda \cap V_{\perp}}=v_{\Lambda} v_{\Lambda *} \cap V_{\|} .
$$

Choosing $\Lambda=\Lambda_{\perp}\left(g^{\prime}\right)$ and using equation (3.17), we now get

$$
v_{\Lambda_{\perp}\left(g^{\prime}\right) \cap V_{\perp}}=v_{\Lambda_{\perp}\left(g^{\prime}\right)} v_{\Lambda_{1}\left(g^{\prime}\right)} .
$$

However,

$$
\Lambda_{\perp}\left(g^{\prime}\right) \bigcap V_{\perp}=\Gamma \bigcap V_{\perp}\left(g^{\prime}\right) \bigcap V_{\perp}=\Gamma \bigcap V_{\perp}=\Lambda_{\perp}
$$

is independent of $g^{\prime}$. Using equations (3.53) and (3.54), we may now reexpress equation (3.50) as

$$
\widetilde{F}(Q, \mu) \simeq \frac{16}{n_{G} v_{\Lambda_{\perp}}} \mathrm{e}^{4 \pi^{2} / \mu}\left(\frac{\mu}{2 \pi}\right)^{12-(1 / 2) k} .
$$

Note that this expression (including its overall normalization) is independent of the charge vector $Q$ irrespective of which twisted sector it arises from. In the specific example of the $Z_{2}$ orbifold model of [23], this feature can be seen explicitly in the results of [7].

We can now try to compute $F(Q, \widehat{N})$ using equation (3.28). The choice of $\epsilon$ in equation (3.28) is arbitrary, but we shall find it convenient to take 
$\epsilon=\mu_{0}$ with $\mu_{0}$ given by the solution of equation (2.17) with $N$ replaced by $\widehat{N}$. Thus,

$$
F(Q, \widehat{N})=\frac{1}{2 \pi i} \int_{\mu_{0}-i \pi}^{\mu_{0}+i \pi} \widetilde{F}(Q, \mu) \mathrm{e}^{\mu \widehat{N}}
$$

We might at this stage be tempted to replace $\widetilde{F}(Q, \mu)$ in this expression by the right-hand side of equation (3.55). However, one needs to exercise a little more care, since the relation (3.55) holds only in the region of small $\mu$, while the integration range over $\mu$ in equation (3.56) extends over a finite range. Thus, replacing $\widetilde{F}(Q, \mu)$ by the right-hand side of equation (3.55) is possible only if we can argue that the dominant contribution to the integral in equation (3.56) comes from a small region around the origin. From, equation (3.55), it follows that $\mu_{0}$ given in equation (2.17), is a saddle point of the integral in equation (3.56) up to exponentially suppressed corrections. Since up to power corrections $\mu_{0} \simeq 2 \pi / \sqrt{\widehat{N}}$, the value of the integrand near this saddle point has a factor of $\mathrm{e}^{4 \pi \sqrt{\widehat{N}}}$, with a factor of $\mathrm{e}^{2 \pi \sqrt{\widehat{N}}}$ coming from the $\widetilde{F}\left(Q, \mu_{0}\right)$ term and another factor of $\mathrm{e}^{2 \pi \sqrt{\widehat{N}}}$ coming from the $\mathrm{e}^{\mu_{0} \widehat{N}}$ term. From the modular properties of $\widetilde{F}(Q, \mu)$, it follows that there are additional divergences of the integrand near the points of the form $\mu=$ $i \nu, \nu=2 \pi p / q$ for integer $p, q$. Near these points, $\widetilde{F}(Q, \mu) \sim \mathrm{e}^{4 \pi^{2} c_{p, q} /(\mu-i \nu)}$ with $c_{p, q}<1$ as long as $\mu$ is in the range $(-i \pi, i \pi) .{ }^{8}$ Since the integration contour has been chosen to have $\operatorname{Re}(\mu)=\mu_{0}$, the maximum contribution to the integrand from a singularity at $\mu=i \nu$ comes when the integration contour passes through the point $\mu=\mu_{0}+i \nu$, and at this point the integrand has a factor of order $\mathrm{e}^{2 \pi \sqrt{\widehat{N}}\left(1+c_{p, q}\right)+i \nu \widehat{N}}$. Since $c_{p, q}<1$, these contributions are exponentially suppressed compared to the contribution $\sim \mathrm{e}^{4 \pi \sqrt{\widehat{N}}}$ from the saddle point at $\mu_{0}$. Thus, we see that up to exponentially suppressed terms, the contribution to the integral comes from a region close to $\mu_{0}$, and hence in the integral appearing in equation (3.56), we can replace $\widetilde{F}(Q, \mu)$ by the right-hand side of equation (3.55) and change the range of integration to be from $\mu_{0}-i a$ to $\mu_{0}+i a$, where $a$ is some small but fixed positive number. This gives

$$
F(Q, \widehat{N}) \simeq \frac{1}{2 \pi i} \frac{16}{n_{G} v_{\Lambda_{\perp}}} \int_{\mu_{0}-i a}^{\mu_{0}+i a} d \mu \mathrm{e}^{4 \pi^{2} / \mu+\mu \widehat{N}}\left(\frac{\mu}{2 \pi}\right)^{12-(1 / 2) k}
$$

\footnotetext{
${ }^{8}$ Due to the $\mu \rightarrow \mu+2 \pi i$ periodicity, at $\mu \simeq \mu_{0} \pm 2 \pi i$, we expect to get back a contribution of strength identical to that near $\mu=\mu_{0}$, but these points are outside the range of integration in equation (3.56).
} 
Using equation (3.24), we now get

$d(Q) \simeq \frac{1}{2 \pi i} \frac{16}{n_{G} v_{\Lambda_{\perp}}} \int_{\mu_{0}-i a}^{\mu_{0}+i a} d \mu \mathrm{e}^{4 \pi^{2} / \mu+\mu N}\left(\frac{\mu}{2 \pi}\right)^{12-(1 / 2) k}, \quad N \equiv \frac{1}{2}\left(\vec{Q}_{\mathrm{R}}^{2}-\vec{Q}_{\mathrm{L}}^{2}\right)$.

This is in precise agreement with the proposed relation (2.16) provided we make the identification

$$
\mathrm{e}^{-C_{0}+C}=\frac{16}{n_{G} v_{\Lambda_{\perp}}}
$$

By the general arguments outlined in section 2, this also establishes the relations (2.24) and (2.26) involving the partition functions and the relation (2.27) involving the entropy.

\section{Reinterpretation of the partition function $\mathcal{F}(\mu)$}

The definition of the partition function $\mathrm{e}^{\mathcal{F}(\mu)}$ corresponds to choosing an ensemble where we introduce a chemical potential $\mu$ conjugate to the combination $N=Q^{2} / 2$. This is somewhat strange since $N$ involves the square of the charge vector and is not additive. It would seem more natural to choose an ensemble where we keep some of the charges fixed and sum over all possible values of the other charges after introducing a chemical potential conjugate to these charges. ${ }^{9}$ In this section, we shall show that due to the Lorentzian signature of the Narain lattice, and the universality of the expression for $d(Q)$, the partition function $\mathrm{e}^{\mathcal{F}(\mu)}$ can also be reinterpreted in this way.

Let us consider a fixed vector $Q_{0}$ in $V_{\perp}$ in the lattice of physical charges, and let $s_{0}$ denote another fixed vector in $V_{\perp}$ which is also in the lattice of physical charges, and which furthermore is null. Then, $Q_{0}+n s_{0}$ for any integer $n$ represents a physical charge, and for this state

$$
N=\frac{1}{2}\left(Q_{0}+n s_{0}\right)^{2}=\frac{1}{2}\left(Q_{0}\right)^{2}+n Q_{0} \cdot s_{0} .
$$

As long as $Q_{0} \cdot s_{0} \neq 0$, we can choose $Q_{0} \cdot s_{0}$ to be positive without any loss of generality. We now introduce an ensemble where we sum over all charges of the form $Q_{0}+n s_{0}$ for fixed $Q_{0}$ and $s_{0}$ by introducing a chemical potential

\footnotetext{
${ }^{9}$ For example, the ensembles used in $[1,7,15]$ is of this type.
} 
$\beta$ conjugate to the variable $n$ :

$$
\exp \left(\widehat{\mathcal{F}}\left(Q_{0}, s_{0}, \beta\right)\right)=\sum_{n} d\left(Q_{0}+n s_{0}\right) \mathrm{e}^{-\beta n} .
$$

Using equation (4.1), this reduces to

$$
\exp \left(\widehat{\mathcal{F}}\left(Q_{0}, s_{0}, \beta\right)\right) \simeq \sum_{n} d_{(1 / 2)\left(Q_{0}\right)^{2}+n Q_{0} \cdot s_{0}} \mathrm{e}^{-\beta n},
$$

where $d_{N}$ as usual denotes the universal formula for the degeneracy of halfBPS elementary string states with $Q^{2}=2 N$. If $Q_{0} \cdot s_{0}=p / q$ for relatively prime integers $p$ and $q$, then we express $n$ as $j q+l$ with $0 \leq l \leq q-1, j \in Z$, and rewrite the sum over $n$ in equation (4.3) as

$$
\begin{aligned}
\exp \left(\widehat{\mathcal{F}}\left(Q_{0}, s_{0}, \beta\right)\right) \\
\simeq \sum_{l=0}^{q-1} \sum_{j \in Z} d_{(1 / 2)\left(Q_{0}\right)^{2}+(j q+l)(p / q)} \mathrm{e}^{-\beta(j q+l)} \\
\quad=\mathrm{e}^{(1 / 2) \beta q Q_{0}^{2} / p} \sum_{l=0}^{q-1} \sum_{j \in Z} d_{(1 / 2)\left(Q_{0}\right)^{2}+l \frac{p}{q}+j p} \mathrm{e}^{-\left((1 / 2)\left(Q_{0}\right)^{2}+l \frac{p}{q}+j p\right) \beta q / p} .
\end{aligned}
$$

Using definition (2.19) of $\mathcal{F}_{\alpha}^{(J)}(\mu)$ and equation (2.25), this may be rewritten as $^{10}$

$$
\begin{aligned}
\exp \left(\widehat{\mathcal{F}}\left(Q_{0}, s_{0}, \beta\right)\right) & \simeq \mathrm{e}^{(1 / 2) \beta q Q_{0}^{2} / p} \sum_{l=0}^{q-1} \frac{1}{p} \exp \left(\mathcal{F}_{(1 / 2)\left(Q_{0}\right)^{2}+l p / q}^{(p)}(\beta q / p)\right) \\
& \simeq \mathrm{e}^{(1 / 2) \beta q Q_{0}^{2} / p} \frac{q}{p} \mathrm{e}^{\mathcal{F}(\beta q / p)} \\
& =\frac{1}{Q_{0} \cdot s_{0}} \mathrm{e}^{\beta Q_{0}^{2} / 2 Q_{0} \cdot s_{0}} \mathrm{e}^{\mathcal{F}\left(\beta / Q_{0} \cdot s_{0}\right)}
\end{aligned}
$$

This gives

$$
\widehat{\mathcal{F}}\left(Q_{0}, s_{0}, \beta\right) \simeq \frac{\beta Q_{0}^{2}}{2 Q_{0} \cdot s_{0}}+\mathcal{F}\left(\frac{\beta}{Q_{0} \cdot s_{0}}\right)-\ln \left(Q_{0} \cdot s_{0}\right) .
$$

This gives a simple relation between the partition function $\mathrm{e}^{\mathcal{F}(\mu)}$ that we have used and the partition function $\mathrm{e}^{\widehat{\mathcal{F}}\left(Q_{0}, s_{0}, \beta\right)}$ defined in equation $(4.2)$. In particular, if we define $\widehat{S}_{\text {stat }}\left(Q_{0}, s_{0}, n\right)$ as the Legendre transform of $\widehat{\mathcal{F}}\left(Q_{0}, s_{0}, \beta\right)$ :

$$
\widehat{S}_{\text {stat }}\left(Q_{0}, s_{0}, n\right)=\widehat{\mathcal{F}}\left(Q_{0}, s_{0}, \beta\right)+\beta n, \quad \frac{\partial \widehat{\mathcal{F}}\left(Q_{0}, s_{0}, \beta\right)}{\partial \beta}+n=0,
$$

\footnotetext{
${ }^{10}$ If the subscript $\frac{1}{2}\left(Q_{0}\right)^{2}+l \frac{p}{q}$ of $\mathcal{F}^{(p)}$ lies outside the range $(-1, p-1)$, then we need to bring it within this range by adding appropriate integral multiples of $p$.
} 
then equation (4.6) implies that $\widehat{S}_{\text {stat }}\left(Q_{0}, s_{0}, n\right)$ is related to the statistical entropy $\widetilde{S}_{\text {stat }}(N)$ defined in equation $(2.28)$ by the simple relation:

$$
\widehat{S}_{\text {stat }}\left(Q_{0}, s_{0}, n\right) \simeq \widetilde{S}_{\text {stat }}\left(N=\frac{1}{2}\left(Q_{0}\right)^{2}+n Q_{0} \cdot s_{0}\right)-\ln \left(Q_{0} \cdot s_{0}\right)
$$

Thus, the two entropies are essentially the same up to an additive factor. This additive factor reflects the difference in step size used in defining the two ensembles. Using equation (4.8), we can now rewrite the conjectured relation $(2.27)$ as

$$
\widehat{S}_{\text {stat }}\left(Q_{0}, s_{0}, n\right) \simeq S_{\mathrm{BH}}\left(N=\frac{1}{2}\left(Q_{0}\right)^{2}+n Q_{0} \cdot s_{0}\right)-C_{0}-\ln \left(Q_{0} \cdot s_{0}\right) .
$$

\section{$5 \quad$ Index or absolute degeneracy?}

For $\mathcal{N}=4$ supersymmetric string theories, one can define an index $\Omega_{4}$ which vanishes for a non-BPS state but is non-zero for half-BPS states [41], and it has been suggested that the computation of the black hole entropy by keeping only the corrections to the generalized prepotential and ignoring other higher derivative corrections might lead to this index rather than the absolute degeneracy [7]. Up to an overall normalization factor, this index counts the number of short multiplets of the supersymmetry algebra weighted by $(-1)^{F}$, where $F$ represents the space-time fermion number of the highest $J_{3}$ state of the supermultiplet, and $J_{3}$ is the third component of the angular momentum of the state. In order to compute this index for fundamental string states, it is more convenient to express $(-1)^{F}$ as $(-1)^{F_{\mathrm{L}}}(-1)^{F_{\mathrm{R}}}$, with $F_{\mathrm{L}}$ and $F_{\mathrm{R}}$ denoting the contribution to the space-time fermion number from the left and the right-handed sector of the world-sheet, respectively. For any string compactification, if we consider half-BPS states which involve excitations of the left-handed oscillators on the world-sheet, then each BPS multiplet can be regarded as a tensor product of a single BPS multiplet representing the ground state of the right-handed oscillators and an arbitrary state involving the left-handed oscillators. $(-1)^{F_{\mathrm{R}}}$ for the highest $J_{3}$ state of such a BPS state is always 1 . Thus, the computation of $\Omega_{4}$ involves computing the trace of $(-1)^{F_{\mathrm{L}}}$ over the BPS states.

For heterotic string compactification, the left-moving sector does not contribute to the fermion number. Hence, each BPS state contributes 1 to $\operatorname{Tr}(-1)^{F_{\mathrm{L}}}$, and $\Omega_{4}$ is simply proportional to the absolute number of BPS states [7]. Thus, in all the formulae we have given in the earlier sections of this paper, e.g., equation (2.18), we can replace the degeneracy $d_{N}$ by the index $\left(\Omega_{4}\right)_{N}$ up to an overall multiplicative factor, and the analysis in the heterotic string theory cannot distinguish these two prescriptions. 
The situation, however, is different in type II string theory. Let us consider, for example, type II string theory compactified on a torus $T^{6}$, and consider a state for which the right-handed oscillators are in the lowest $L_{0}$ eigenvalue state consistent with GSO projection. In this case, at any given level, there are equal number of states in the left-hand sector with $(-1)^{F_{\mathrm{L}}}=1$ and $(-1)^{F_{\mathrm{L}}}=-1$. As a result, the index $\Omega_{4}$ vanishes. This is encouraging since it is known that for type II superstring theory on a torus, inclusion of higher derivative corrections to the generalized prepotential does not produce a finite area for the black hole representing the fundamental type II string. Thus, if in our formulae, we replace the degeneracy $d_{N}$ by the index $\left(\Omega_{4}\right)_{N}$, then the results agree trivially since the leading contribution to both side of various formulae vanishes.

This, however, is not the end of the story. Consider, for example, type IIA string theory on $T^{4} \times \widetilde{S}^{1} \times S^{1}$ and take the quotient of this theory by a $Z_{2}$ group that acts as $(-1)^{F_{\mathrm{L}}}$ together with a half-shift along $\widetilde{S}^{1}$ [50]. In this case, if we consider an untwisted sector state that carries even unit of momentum along $\widetilde{S}^{1}$, then the $(-1)^{F_{\mathrm{L}}}$ for the surviving states must be 1 , and as a result, when we evaluate $\operatorname{Tr}(-1)^{F_{\mathrm{L}}}$ over BPS supermultiplets of this type, we simply get the total number of BPS states in this sector. On the other hand, for states carrying odd units of winding along $\widetilde{S}^{1}$, the $(-1)^{F_{\mathrm{L}}}$ quantum number must be -1 , and as a result, $\operatorname{Tr}(-1)^{F_{\mathrm{L}}}$ over such BPS supermultiplets gives an answer that is negative of the total number of BPS states in that sector. Thus, $\Omega_{4}$ is non-zero for both sectors. Black hole entropy for this system will continue to vanish, however, due to an argument of [12] that shows that the leading term in the expression for black hole entropy has a universal form independent of compactification and hence vanishing of the entropy of the black hole representing fundamental string in type II string theory on $T^{6}$ automatically implies the vanishing of the black hole entropy in this new asymmetric orbifold. Thus, we see that there is a mismatch between $\Omega_{4}$ and $\mathrm{e}^{S_{\mathrm{BH}}}$ even at the leading order.

The exact interpretation of this discrepancy is not completely clear to us. We note, however, that unlike the heterotic case, where for a given charge $Q$ the total number of BPS states and hence also the index $\Omega_{4}$ is a function of the combination $N=Q^{2} / 2$ only up to exponentially suppressed terms, here $\Omega_{4}$ can take different values for different charges even if $Q^{2}$ is the same for these charges. Up to exponentially suppressed corrections, these values differ from each other by a - sign. Thus, if we replace $d_{N}$ by $\left(\Omega_{4}\right)_{N}$ in equation (2.18), we no longer have a well-defined expression; the result depends on the representative state that we use to compute $\left(\Omega_{4}\right)_{N}$. This could be the reason why the correspondence between black hole entropy and $\Omega_{4}$ of BPS states does not hold in this case. 


\section{Acknowledgement}

I wish to thank A. Dabholkar, R. Gopakumar, D. Jatkar, S. Parvizi, J. Samuel, D. Surya Ramana, and A. Tavanfar for useful discussions.

\section{References}

[1] A. Dabholkar, Exact counting of black hole microstates, hepth/0409148.

[2] A. Dabholkar, R. Kallosh and A. Maloney, A stringy cloak for a classical singularity, hep-th/0410076.

[3] A. Sen, How does a fundamental string stretch its horizon?, hepth/0411255.

[4] V. Hubeny, A. Maloney and M. Rangamani, String-corrected black holes, hep-th/0411272.

[5] D. Bak, S. Kim and S.J. Rey, Exactly soluble BPS black holes in higher curvature $N=2$ supergravity, hep-th/0501014.

[6] A. Sen, Black holes, elementary strings and holomorphic anomaly, hepth/0502126.

[7] A. Dabholkar, F. Denef, G.W. Moore and B. Pioline, Exact and asymptotic degeneracies of small black holes, hep-th/0502157.

[8] A. Dabholkar and J.A. Harvey, Nonrenormalization of the Superstring Tension, Phys. Rev. Lett. 63 (1989), 478.

[9] A. Dabholkar, G.W. Gibbons, J.A. Harvey and F. Ruiz Ruiz, Superstrings and Solitons, Nucl. Phys. B 340 (1990), 33.

[10] A. Sen, Extremal black holes and elementary string states, Mod. Phys. Lett. A 10 (1995), 2081; hep-th/9504147.

[11] A.W. Peet, Entropy and supersymmetry of D-dimensional extremal electric black holes versus string states, Nucl. Phys. B 456 (1995), 732; hep-th/9506200.

[12] A. Sen, Black holes and elementary string states in $N=2$ supersymmetric string theories, J. High Energy Phys. 9802 (1998), 011; hepth/9712150.

[13] A. Strominger and C. Vafa, Microscopic Origin of the BekensteinHawking Entropy, Phys. Lett. B 379 (1996), 99; hep-th/9601029.

[14] J.M. Maldacena, A. Strominger and E. Witten, Black hole entropy in M-theory, J. High Energy Phys. 9712 (1997), 002; hep-th/9711053.

[15] H. Ooguri, A. Strominger and C. Vafa, Black hole attractors and the topological string, hep-th/0405146. 
[16] E. Verlinde, Attractors and the holomorphic anomaly, hep-th/0412139.

[17] M. Bershadsky, S. Cecotti, H. Ooguri and C. Vafa, Holomorphic anomalies in topological field theories, Nucl. Phys. B 405 (1993) 279; hep-th/9302103.

[18] I. Antoniadis, E. Gava, K.S. Narain and T.R. Taylor, Topological amplitudes in string theory, Nucl. Phys. B 413 (1994), 162; hep-th/9307158.

[19] M. Bershadsky, S. Cecotti, H. Ooguri and C. Vafa, Kodaira-Spencer theory of gravity and exact results for quantum string amplitudes, Commun. Math. Phys. 165 (1994), 311; hep-th/9309140.

[20] G. Lopes Cardoso, B. de Wit and T. Mohaupt, Macroscopic entropy formulae and non-holomorphic corrections for supersymmetric black holes, Nucl. Phys. B 567 (2000), 87; hep-th/9906094.

[21] G.L. Cardoso, B. de Wit, J. Kappeli and T. Mohaupt, Asymptotic degeneracy of dyonic $N=4$ string states and black hole entropy, hepth/0412287.

[22] S. Chaudhuri, G. Hockney and J.D. Lykken, Maximally supersymmetric string theories in $D<10$, Phys. Rev. Lett. 75 (1995), 2264; hepth/9505054.

[23] S. Chaudhuri and J. Polchinski, Moduli space of CHL strings, Phys. Rev. D 52 (1995), 7168; hep-th/9506048.

[24] J.H. Schwarz and A. Sen, Type IIA dual of the six-dimensional CHL compactification, Phys. Lett. B 357 (1995), 323; hep-th/9507027.

[25] C. Vafa and E. Witten, Dual string pairs with $N=1$ and $N=2$ supersymmetry in four dimensions, Nucl. Phys. Proc. Suppl. 46 (1996), 225; hep-th/9507050.

[26] S. Chaudhuri and D.A. Lowe, Type IIA heterotic duals with maximal supersymmetry, Nucl. Phys. B 459 (1996), 113; hep-th/9508144.

[27] P.S. Aspinwall, Some relationships between dualities in string theory, Nucl. Phys. Proc. Suppl. 46 (1996), 30; hep-th/9508154.

[28] S. Ferrara, R. Kallosh and A. Strominger, $N=2$ extremal black holes, Phys. Rev. D 52 (1995), 5412; hep-th/9508072.

[29] A. Strominger, Macroscopic Entropy of $N=2$ Extremal Black Holes, Phys. Lett. B 383 (1996), 39; hep-th/9602111.

[30] S. Ferrara and R. Kallosh, Supersymmetry and Attractors, Phys. Rev. D 54 (1996), 1514; hep-th/9602136.

[31] B. de Wit, $N=2$ electric-magnetic duality in a chiral background, Nucl. Phys. Proc. Suppl. 49 (1996), 191; hep-th/9602060.

[32] B. de Wit, $N=2$ symplectic reparametrizations in a chiral background, Fortsch. Phys. 44 (1996), 529; hep-th/9603191. 
[33] G. Lopes Cardoso, B. de Wit and T. Mohaupt, Corrections to macroscopic supersymmetric black-hole entropy, Phys. Lett. B 451 (1999), 309; hep-th/9812082.

[34] G. Lopes Cardoso, B. de Wit and T. Mohaupt, Deviations from the area law for supersymmetric black holes, Fortsch. Phys. 48 (2000), 49; hep-th/9904005.

[35] G. Lopes Cardoso, B. de Wit and T. Mohaupt, Area law corrections from state counting and supergravity, Class. Quant. Grav. 17 (2000), 1007; hep-th/9910179.

[36] T. Mohaupt, Black hole entropy, special geometry and strings, Fortsch. Phys. 49 (2001), 3; hep-th/0007195.

[37] G. Lopes Cardoso, B. de Wit, J. Kappeli and T. Mohaupt, Stationary BPS solutions in $N=2$ supergravity with $\mathrm{R}^{2}$ interactions, J. High Energy Phys. 0012 (2000), 019; hep-th/0009234.

[38] G.L. Cardoso, B. de Wit, J. Kappeli and T. Mohaupt, Examples of stationary BPS solutions in $N=2$ supergravity theories with $\mathrm{R}^{2}$ interactions, Fortsch. Phys. 49 (2001), 557; hep-th/0012232.

[39] B. Zwiebach, Curvature Squared Terms and String Theories, Phys. Lett. B 156 (1985), 315.

[40] J.A. Harvey and G.W. Moore, Fivebrane instantons and $\mathrm{R}^{2}$ couplings in $N=4$ string theory, Phys. Rev. D 57 (1998), 2323; hep-th/9610237.

[41] A. Gregori, E. Kiritsis, C. Kounnas, N.A. Obers, P.M. Petropoulos and $\mathrm{B}$. Pioline, $\mathrm{R}^{2}$ corrections and non-perturbative dualities of $N=4$ string ground states, Nucl. Phys. B 510 (1998), 423; hepth/9708062.

[42] R.M. Wald, Black hole entropy in the Noether charge, Phys. Rev. D 48 (1993), 3427; gr-qc/9307038.

[43] T. Jacobson, G. Kang and R.C. Myers, On black hole entropy, Phys. Rev. D 49 (1994), 6587; gr-qc/9312023.

[44] V. Iyer and R.M. Wald, Some properties of Noether charge and a proposal for dynamical black hole entropy, Phys. Rev. D 50 (1994), 846; gr-qc/9403028.

[45] T. Jacobson, G. Kang and R.C. Myers, Black hole entropy in higher curvature gravity, gr-qc/9502009.

[46] K.S. Narain, New Heterotic String Theories in Uncompactified Dimensions < 10, Phys. Lett. B 169 (1986), 41.

[47] K.S. Narain, M.H. Sarmadi and E. Witten, A Note on Toroidal Compactification of Heterotic String Theory, Nucl. Phys. B 279 (1987), 369. 
[48] K.S. Narain, M.H. Sarmadi and C. Vafa, Asymmetric Orbifolds: Path Integral and Operator Formulations, Nucl. Phys. B 356 (1991), 163.

[49] J. Martinet, Perfect Lattices in Euclidean Spaces, Springer-Verlag, Berlin, 2003, 9.

[50] A. Sen and C. Vafa, Dual pairs of type II string compactification, Nucl. Phys. B 455 (1995), 165; hep-th/9508064. 